\title{
Catalytic Wet Oxidation of Pharmaceutical Sludge by Molecular Sieve Loaded with $\mathrm{Cu} / \mathrm{Ce}$
}

\author{
Xu Zeng ${ }^{1, *}$, Jun Liu ${ }^{1,2}$ and Jianfu Zhao ${ }^{1}$ \\ 1 State Key Laboratory of Pollution Control and Resources Reuse, College of Environmental Science and \\ Engineering, Tongji University, 1239 Siping Road, Shanghai 200092, China; robinmark88@sina.com (J.L.); \\ 15523@tongji.edu.cn (J.Z.) \\ 2 Shanghai Electric Power Generatoin Environment Protection Engineering Co., Ltd., Shanghai 201612, China \\ * Correspondence: zengxu@tongji.edu.cn; Tel.: +86-021-65-986-295
}

Received: 24 December 2017; Accepted: 29 January 2018; Published: 8 February 2018

\begin{abstract}
In the present study, the catalytic wet oxidation of pharmaceutical sludge by molecular sieve loaded with $\mathrm{Cu} / \mathrm{Ce}$ as catalyst was investigated. Experiments were performed in an experimental batch reactor. Reaction parameters including catalyst dose, temperature, time, and oxygen pressure were discussed. The results showed that the catalysts prepared by co-precipitating method have good catalytic performance. Under optimum conditions, the highest volatile suspended solids (VSS) removal rate approximately $92 \%$ and chemical oxygen demand (COD) removal rate of $75 \%$ were obtained at $260^{\circ} \mathrm{C}$ for $60 \mathrm{~min}$ with initial oxygen pressure $1.0 \mathrm{MPa}$ and the catalyst $10 \mathrm{~g} / \mathrm{L}$. These results implied that the catalytic wet oxidation of pharmaceutical sludge by molecular sieve loaded with $\mathrm{Cu} / \mathrm{Ce}$ is a promising method for the highly efficient degradation of the sludge.
\end{abstract}

Keywords: pharmaceutical sludge; wet oxidation; molecular sieve; chemical oxygen demand; volatile suspended solids

\section{Introduction}

Large amounts of pharmaceutical sludge were been generated due to the fast development of pharmaceutical industry. The treatment of pharmaceutical sludge has become a growing concern, because non-degradable organic pollutants, especially highly toxic organic pollutants and heavy metals were contained in the sludge. The effective removal of such pollutants is a challenging task. Biotechnology, adsorption, and incineration have been traditionally applied for the treatment of pharmaceutical sludge. However, toxic pollutants are lethal to the microorganisms in bioprocesses. In addition, adsorption and incineration merely transfer pollutants from a liquid to a solid or to air, leaving a contaminated adsorbent or combustion by-products for further disposal. Therefore, alternative technologies that can deal with the highly toxic and non-biodegradable pollutants are strongly desired.

Wet Oxidation (WO) is one of the numerous technologies that have been studied for sludge minimization and treatment, which were conducted under the temperature $150-320^{\circ} \mathrm{C}$ and pressure 20-150 bar [1]. In WO process, organic pollutants are partially converted to carbon dioxide, water and inorganic salts, avoiding the generation of harmful emissions. During the wet oxidation of sludge, a large proportion of insoluble organics are first solubilised through hydrolytic depolymerization reactions, which are the most important oxidising species together with the formation of free radicals. Subsequent oxidative reactions convert these hydrolysis products into increasingly simpler organics such as organic acids and alcohols. Finally, these products can be further oxidized [2]. Organic acids (mainly acetic acid) are the major intermediate products of sludge [3]. Recently, the production of these acids has received increasing attention. Strong et al. examined destruction of municipal sludge and production of organic acids using wet oxidation treatment followed by anaerobic fermentation [4]. 
These studies demonstrated combined techniques and new approaches for the handling of municipal sludge. Because there are almost no harmful materials or secondary pollution to the environment, this process is a green and environmental friendly technology. In addition, it avoids the need for the energy-intensive dewatering step, and can directly process liquid and slurry sludge. Therefore, WO has a high potential for the treatment of sludge.

Catalytic wet air oxidation (CWAO) has been shown to be an effective and environmentally friendly way which can decrease the operating conditions of $\mathrm{WO}$, enhances the reaction rate, and shortens the residence time. The use of catalysts makes the process more attractive by achieving high conversion at a considerably lower temperature and lower pressure. These catalysts exhibit the advantages of a heterogeneously catalyzed process. Unlike a homogeneous catalyst they have relatively higher oxidation efficiency and lower sensitivity to $\mathrm{pH}$ under equal reaction conditions. The preparation of efficient and durable catalysts is still a challenge. Molecular sieves when supported onto another functional material were exhibited high potential due to their high thermal stability, their relatively high specific surface area and/or their beneficial role in terms of resistance to poisons $[5,6]$. Many materials containing mainly metals as the precursors supported/intercalated on/in molecular sieves are proposed as catalysts for the oxidation of organic compounds $[7,8]$. During the past decades, transition metal modified mesoporous silicates have been studied widely due to the excellent acidity or oxidizing property [9]. Li et al. reported that metal ions modified molecular sieves $(\mathrm{Co}, \mathrm{Ce}, \mathrm{Cu})$ improved the oxidation of acetaldehyde [10]. Chou et al. reported that $\mathrm{Cu}$ (II)-substituted molecular sieves were efficient in liquid phase oxidation of aromatic compounds [11]. Taran et al. studied the peroxide oxidation of formic acid and phenol by copper- and iron-containing ZSM-5-30 [12]. They reported that copper-containing catalysts were more active for formic acid oxidation than the iron-containing catalyst. From the literature, it was realized that modified molecular sieves are good candidates for the oxidation reactions.

In this study, the catalytic wet oxidation of pharmaceutical sludge by molecular sieve loaded with $\mathrm{Cu} / \mathrm{Ce}$ was investigated. The effects of reaction parameters were discussed, including the additional amount of catalyst, reaction temperature, time and initial oxygen pressure. Particularly, in case of pharmaceutical sludge, which is a complex and heterogeneous matrix, a great number of compounds are involved into the process. Therefore, as an alternative approach to a detailed microscale description, certain lumped parameters, such as COD or VSS removal rates, can be profitably used for the assessment. The goal of this work is to assess the degradation efficiency of pharmaceutical sludge by catalytic wet oxidation.

\section{Materials and Methods}

\subsection{Materials}

The molecular sieve catalyst used in this study was purchased from Shanghai Shanpu Chemical Co. Ltd. (Shanghai, China). The diameter of the catalyst was about $3 \mathrm{~mm}$, and the molecular formula was $\mathrm{Na}_{12}\left[\left(\mathrm{AlO}_{2}\right)_{12}\left(\mathrm{SiO}_{2}\right)_{12}\right] \cdot \mathrm{xH}_{2} \mathrm{O}$. Other materials, such as $\mathrm{Cu}\left(\mathrm{NO}_{3}\right)_{2}, \mathrm{Ce}\left(\mathrm{NO}_{3}\right)_{3}$ and $\mathrm{NaOH}$, were purchased from Shanghai Sinopharm Chemical Reagent, Shanghai, China). The real pharmaceutical sludge was collected from a synthetic pharmaceutical factory. The characters of the raw sludge are as follows: COD 18,000 19,000 mg/L, pH 7.5 8.0, suspended solids 40 45 g/L. The sludge was adopted for the experiments without any treatment.

\subsection{WO Reaction System}

All experiments in this study were performed in a $100 \mathrm{~mL}$ SUS316 autoclave reactor equipped with a mechanically driven stirrer, as shown in Figure 1. The reactor was purchased from Anhui Kemi Machinery Technology Co. Ltd., Hefei, China. Temperature was modified in the range of $180-260{ }^{\circ} \mathrm{C}$ using an electric jacket, and the initial oxygen partial pressure was varied in the range of 0.2 1.0 MPa. Stirring speed was $300 \mathrm{rpm} / \mathrm{min}$. Once the desired temperature was reached, this moment is taken as 
zero time of the reaction time. The experimental procedure is as follows: $50 \mathrm{~mL}$ of pharmaceutical sludge solution and a certain amount catalyst were put into the reactor, then the reactor was heated to expected temperature. After the desired reaction time, the reactor was removed from the oven and allowed to cool to room temperature. The reaction pressure corresponded to the self-pressurization with saturated vapour pressure.

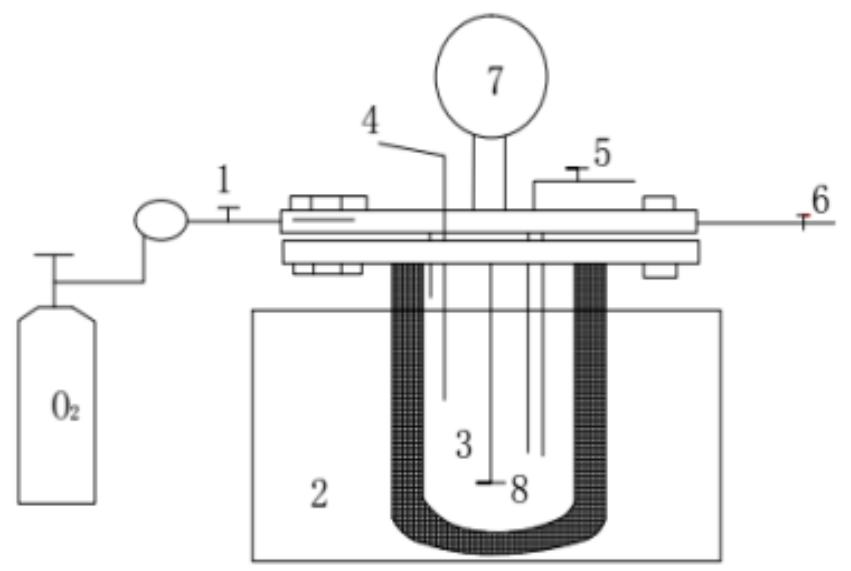

Figure 1. Diagram of wet oxidation reactor.1: Oxygen supply; 2: Heating jacket; 3: Oxidation reactor; 4 : Thermocouple; 5: Discharge of gas; 6: Bleeder valve; 7: Pressure meter; 8: Stirrer.

\subsection{Synthesis of Catalyst}

The $\mathrm{Cu}$-loaded, Ce-loaded and $(\mathrm{Cu}, \mathrm{Ce})$-loaded catalysts were prepared by loading metal ion on the molecular sieve using $0.1 \mathrm{~mol} \cdot \mathrm{L}^{-1} \mathrm{Cu}\left(\mathrm{NO}_{3}\right)_{2}$ and $\mathrm{Ce}\left(\mathrm{NO}_{3}\right)_{3}$ solution. The prepared solids were washed withde-ionized water for three times, then it was dried at $120^{\circ} \mathrm{C}$ overnight and calcined at $450{ }^{\circ} \mathrm{C}$ for $4 \mathrm{~h}$. The X-ray diffraction (XRD) analysis of the prepared catalysts was performed. The results showed that the chemical form of $\mathrm{Ce}$ and $\mathrm{Cu}$ in the catalyst were oxides. The quantity of $\mathrm{Cu}$ and Ce oxides in the catalyst was $5 \%$, which means that the $(\mathrm{Cu}, \mathrm{Ce})$-loaded catalyst was synthesized successfully. Then we compared the catalytic activity of the $\mathrm{Cu}$-loaded, $\mathrm{Ce}$-loaded, and (Cu,Ce)-loaded catalysts by the catalytic wet oxidation of pharmaceutical sludge experiments. The results showed that the catalytic activity of the $\mathrm{Cu}$-loaded catalyst was the best. The $(\mathrm{Cu}, \mathrm{Ce})$-loaded catalyst was better than the Ce-loaded catalyst. Besides, we compared the three kinds of catalyst. During the catalytic wet oxidation, about $5 \mathrm{mg} / \mathrm{L}$ of cupper was leached from the catalyst. However, the catalytic stability of Ce-loaded is better. According these comparative experiments, we use bimetallic catalyst, i.e., $(\mathrm{Cu}, \mathrm{Ce})$-loaded catalyst, instead of $\mathrm{Cu}$-loaded or Ce-loaded catalyst.

\subsection{Analysis}

XRD analysis of the catalysts was performed by using a Bruker D8 Advance X-ray diffractometer equipped with $\mathrm{Cu} K \alpha$ radiation $\left(\lambda=1.5406 \AA\right.$ ), employing a scanning rate of 0.2 degree $\mathrm{s}^{-1}$ and with $2 \theta$ ranging from $10^{\circ}$ to $90^{\circ}$. The accelerating voltage was set at $40 \mathrm{kV}$ with a $40 \mathrm{~mA}$ flux. Quantitative phase analysis used Rietveld method, which was performed by the software TOPAS 4.2 from Bruker AXS GmbH, Karlsruhe, Germany [13]. This software is based on the fundamental parameter approach (FPA), which considers the geometric and unit-specific parameters [14]. COD and VSS removal rates of the mixed liquor were used to assess treatment efficiency. COD was measured by the potassium dichromate oxidation method (Hach Heating System, Hach Corporation, Loveland, CO, USA). VSS was measured by the Ignition loss method. $\mathrm{pH}$ was measured with a $\mathrm{pH}$ metre ( $\mathrm{pH}-201$, Hanna Corporation, Padova, Italy). 


\section{Results and Discussion}

\subsection{Effect of Catalyst Amount on the Sludge Degradation}

To assess the effect of catalyst on the sludge degradation, experiments were performed with different amounts of catalyst, from 0 to $1.0 \mathrm{~g}$ at $220^{\circ} \mathrm{C}$ for $60 \mathrm{~min}$ with initial oxygen pressure of 1.0 MPa. The results can be seen in Figure 2. As shown in Figure 2, the COD and VSS removal rates increased with the increase of catalyst amount. The prepared catalyst had a remarkable catalytic effect. The highest VSS rate, approximately $80 \%$, was obtained with $1.0 \mathrm{~g}$ of catalyst. The COD removal rate was approximately $60 \%$. The VSS removal rate was approximately $70 \%$, even with no catalyst. The reason may be that the sludge was dissolved to the water under high temperature conditions. However, the COD removal rate was not very high even with enough oxygen gas. It has been reported extensively that acetic acid was produced in the WO treatment process, and acetic acid is not easily oxidized to $\mathrm{CO}_{2}$ and $\mathrm{H}_{2} \mathrm{O}$. The results suggest the presence of a significant amount of refractory compound (e.g., acetic acid) during the WO treatment process. Therefore, the COD removal rate was not very high. These results illustrated that the prepared catalyst was suitable for the catalytic wet oxidation of pharmaceutical sludge.

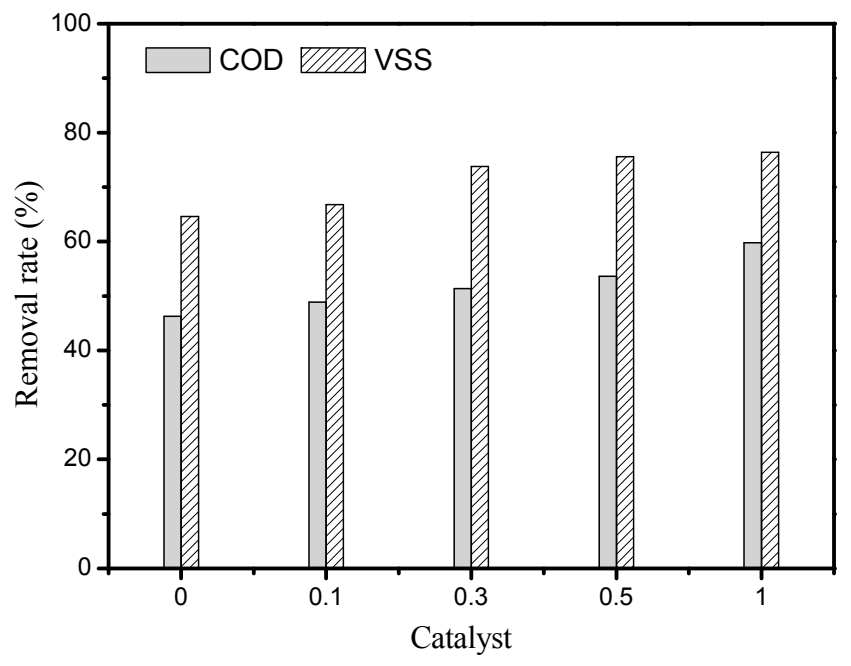

Figure 2. Effect of catalyst amount on the COD and VSS removal rates (Time: $60 \mathrm{~min}$; Temp.: $220{ }^{\circ} \mathrm{C}$; Oxygen dosage: $1.0 \mathrm{MPa})$.

\subsection{Effect of Temperature on the Sludge Degradation}

Temperature is considered to be an important parameter in the wet oxidation process. According to Arrhenius' law [15], higher reaction efficiency would be obtained with higher reaction temperature. Above $100^{\circ} \mathrm{C}$, the oxygen solubility increases with temperature. For the CWAO of sludge, due to the exothermic phenomenon, a slight change could bring about an impact on the reaction results. The activation energy of some organic matter is lower, the reaction efficiency could be very high once the exothermal process provides the required heat [16]. The effect of reaction temperature (in the range of $180-260{ }^{\circ} \mathrm{C}$ ) on the COD and VSS removal rate with reaction time 60 min, oxygen pressure 1.0 MPa, and catalyst $0.5 \mathrm{~g}$ was investigated. The results can be seen in Figure 3 . As expected, from the Arrhenius law in the kinetic regime, the oxidation reaction, in terms of COD degradation and the VSS removal rate, accelerates with increasing temperature. The COD removal rate is relatively low at the reaction temperature of $180^{\circ} \mathrm{C}$. Theoretically, the COD removal rate can be very high at high reaction temperatures with enough oxidizing agents. However, with the increase of temperature from $180{ }^{\circ} \mathrm{C}$ to $260{ }^{\circ} \mathrm{C}$, the VSS removal rate rose to $90 \%$ with the COD removal rate remaining at $80 \%$. Probably because of the incomplete degradation of COD, even the organic pollutants in sludge 
were almost oxidized completely. It has been reported that the products of wet oxidation of organics were mainly carboxylic acids with small molecule weights, such as formic acid or acetic acid, which were not easily oxidized under hydrothermal conditions. From the above results, we concluded that higher temperatures were favorable for the catalytic WO of pharmaceutical sludge. However, from the practical point of view, higher temperatures lead to higher operating costs and more severe corrosion problems. Therefore, runs when other operating parameters were discussed, the reaction temperature of $220^{\circ} \mathrm{C}$ was adopted in the following.

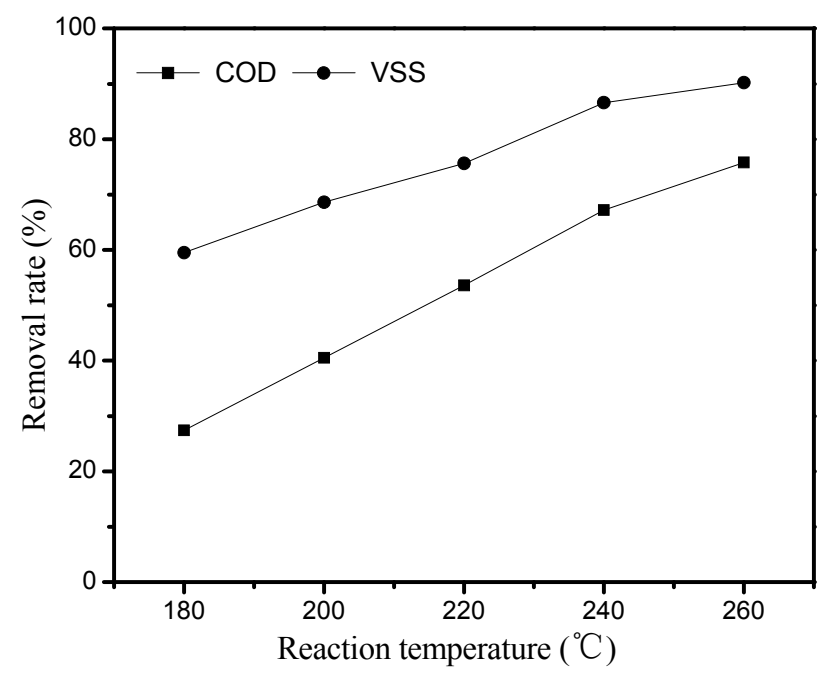

Figure 3. Effect of temperature on the COD and VSS removal rates (Time: 60 min; Catalyst: $0.5 \mathrm{~g}$; Oxygen dosage: $1.0 \mathrm{MPa}$ ).

\subsection{Effect of Reaction Time on the Sludge Degradation}

Figure 4 shows the effect of time on the COD and VSS removal rates at $220{ }^{\circ} \mathrm{C}$ with an initial oxygen pressure at $1.0 \mathrm{MPa}$. The reaction time was changed from $20 \mathrm{~min}$ to $60 \mathrm{~min}$. As shown in Figure 4 , the results indicate that reaction time has a certain effect on sludge treatment. With the extension of time, the removal rates gradually increased. The results show that the COD and VSS removal rates are very high, even with the reaction time of $20 \mathrm{~min}$, which means that the wet oxidation process took place very quickly. This behaviour was expected because the free radical reaction was very fast. It has been widely acknowledged that $\mathrm{OH}$ is the most active species in wet oxidation system. In Ricq's study, the reaction mechanism of the degradation of sewage sludge by wet air oxidation was studied [17]. The study of the use of cellulose as a model compound for activated sludge confirms optimization results and leads to the identification of by-products and free radical species participating in the reaction. With the extension of time, the degradable organic in liquid accumulated. Organic matter of higher activation energy began to oxidize, so that the overall reaction rate was greatly improved. The COD and VSS removal rate increased gradually with the increase of reaction time, and the change trends were similar. These phenomena indicate that the solid phase organic matter was transferred into the liquid phase within a short time, which leads to a high VSS removal rate. However, the COD removal rate was not high. Considering the oxidation efficiency and economic factors, we chose $60 \mathrm{~min}$ as the reaction time for the subsequent study of wet oxidation. 


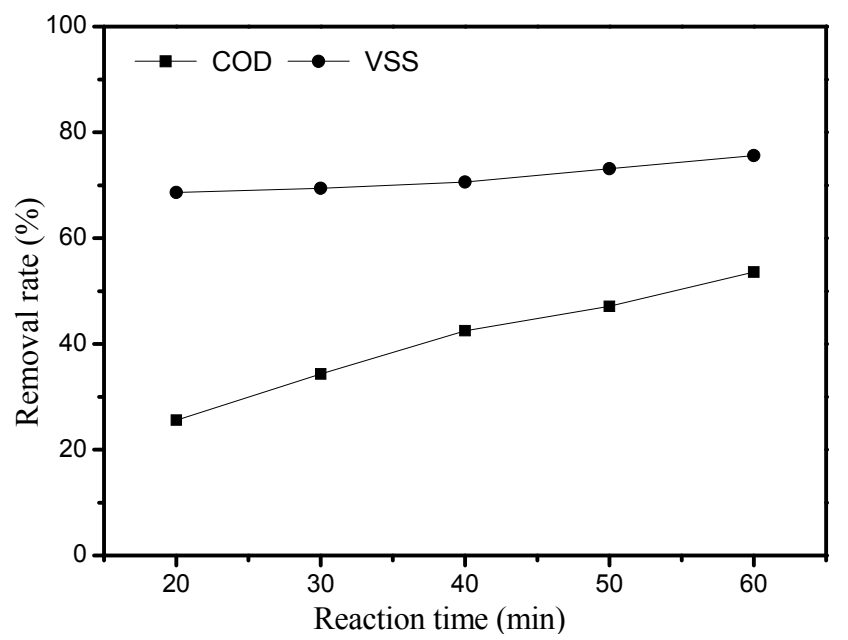

Figure 4. Effect of reaction time on the COD and VSS removal rates (Temp.: $220^{\circ} \mathrm{C}$; Catalyst: 0.5 g; Oxygen dosage: $1.0 \mathrm{MPa}$ ).

\subsection{Effect of Initial Oxygen Pressure on the Sludge Degradation}

To gain better insight into the effect of oxygen pressure on COD and VSS removal rates, experiments were conducted under oxygen pressures varying from 0.2 to $1.0 \mathrm{MPa}$. The results are shown in Figure 5. It can be seen that oxygen pressure plays an important role in COD and VSS removal. It is found that COD removal increases with increasing oxygen pressure. This behaviour was expected because the increase of the oxidant concentration usually leads to an increase in the oxidation rate. These results indicate that high oxygen pressures effectively eliminate refractory and toxic organic compounds and yield high COD and VSS conversions in the WAO process. Under high oxygen pressures, the amount of dissolved oxygen increases in the solution, which can be helpful for the formation of strong oxidation species. However, the COD and VSS removal rate changed little with the increase of oxygen pressure from 0.8 to $1.0 \mathrm{MPa}$. The reason may be that some by-products formed during the wet oxidation are non-oxidizable under the employed conditions because a plateau is observed in the oxygen pressure range from 0.8 to $1.0 \mathrm{MPa}$. These results indicate that the amount of oxidizing agent is enough for the wet oxidation.

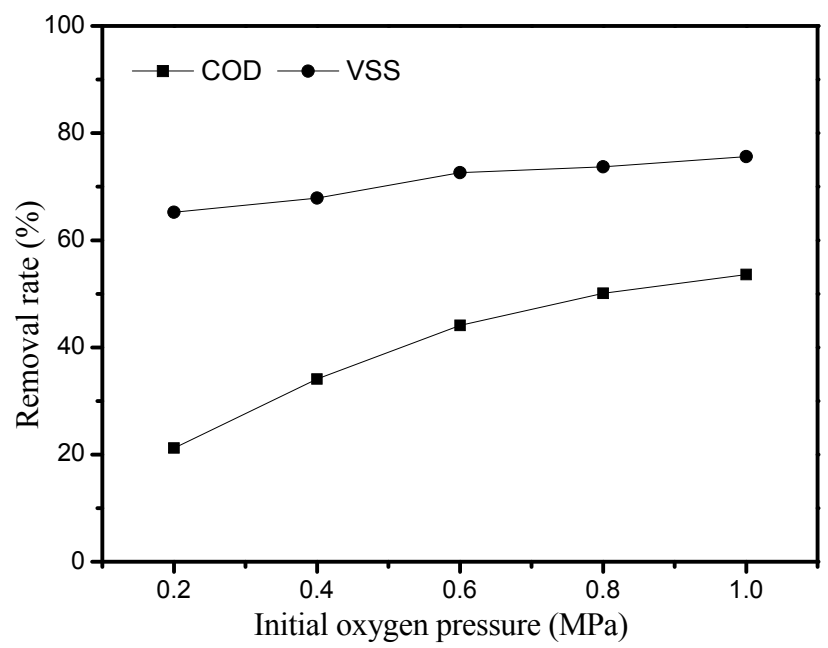

Figure 5. Effect of oxygen dosage on the COD and VSS removal rates (Time: 60 min; Temp.: $220^{\circ} \mathrm{C}$; Catalyst: $0.5 \mathrm{~g})$. 


\section{Conclusions}

In the present study, the catalytic wet oxidation of pharmaceutical sludge by molecular sieve loaded with $\mathrm{Cu} / \mathrm{Ce}$ as catalyst was investigated. Experiments were performed in an experimental batch reactor. The results showed that the catalyst prepared by co-precipitating with nitrate has good catalytic performance. The prepared catalyst had a remarkable catalytic effect, and the COD and VSS removal rates increased with the increase of catalyst amount. Based on the discussion of reaction parameters, including catalyst dose, temperature, time, and oxygen pressure, VSS removal rate approximately $92 \%$ and COD removal rate $75 \%$ were obtained at $260{ }^{\circ} \mathrm{C}$ for 60 min with initial oxygen pressure $1.0 \mathrm{MPa}$ and the catalyst $10 \mathrm{~g} / \mathrm{L}$. These results implied that catalytic wet oxidation of pharmaceutical sludge by molecular sieve loaded with $\mathrm{Cu} / \mathrm{Ce}$ is a promising method for the highly efficient degradation of the sludge.

Acknowledgments: The authors gratefully acknowledge financial support from Shanghai Pujiang Program, China (No. 16PJ1432900).

Author Contributions: Xu Zeng and Jianfu Zhao conceived and designed the experiments; Jun Liu performed the experiments; Jun Liu and Xu Zeng analyzed the data; $X \mathrm{u}$ Zeng contributed reagents/materials/analysis tools; $\mathrm{Xu}$ Zeng wrote the paper.

Conflicts of Interest: The authors declare no conflict of interest.

\section{References}

1. Pike, P.; Wilson, D.I.; Baroutian, S.; Andrews, J.; Gapes, D.J. A kinetic model of municipal sludge degradation during non-catalytic wet oxidation. Water Res. 2015, 87, 225-236. [CrossRef] [PubMed]

2. Robert, R.; Barbati, S.; Ricq, N.; Ambrosio, M. Intermediates in wet oxidation of cellulose: Identification of hydroxyl radical and characterization of hydrogen peroxide. Water Res. 2002, 36, 4821-4829. [CrossRef]

3. Saeid, B.; Daniel, J.G.; Ajit, K.S.; Mohammed, M.F.; Brent, R.Y. Formation and degradation of valuable intermediate products during wet oxidation of municipal sludge. Bioresour. Technol. 2016, 205, 280-285.

4. Strong, P.J.; McDonald, B.; Gapes, D.J. Combined thermochemical and fermentative destruction of municipal biosolids: A comparison between thermal hydrolysis and wet oxidative pre-treatment. Bioresour. Technol. 2011, 102, 5520-5526. [CrossRef] [PubMed]

5. Reddy, B.M.; Saikia, P.; Bharali, P. Highly dispersed $\mathrm{Ce}_{x} \mathrm{Zr}_{1-\mathrm{x}} \mathrm{O}_{2}$ nano-oxides over alumina, silica and titania supports for catalytic applications. Catal. Surv. Asia 2008, 12, 214-228. [CrossRef]

6. Moretti, E.; Lenarda, M.; Storaro, L.; Talon, A.; Montanari, T.; Busca, G.; Rodríguez-Castellón, E.; Jiménez-López, A.; Turco, M.; Bagnasco, G.; et al. One-step synthesis of a structurally organized mesoporous $\mathrm{CuO}-\mathrm{CeO}_{2} / \mathrm{Al}_{2} \mathrm{O}_{3}$ system for the preferential $\mathrm{CO}$ oxidation. Appl. Catal. A 2008, 335, 46-55. [CrossRef]

7. Hu, X.; Lam, F.; Cheung, L.; Chan, K.; Hao, X.; Lu, G. Copper/MCM-41 as catalyst for photochemically enhanced oxidation of phenol by hydrogen peroxide. Catal. Today 2001, 68, 129-133. [CrossRef]

8. Parvulescu, V.; Su, B.L. Iron, cobalt or nickel substituted MCM-41 molecular sieves for oxidation of hydrocarbons. Catal. Today 2001, 69, 315-322. [CrossRef]

9. Zhan, W.Q.; Guo, Y.L.; Wang, Y.Q.; Liu, X.H.; Guo, Y.; Wang, Y.S.; Zhang, Z.G.; Lu, G.Z. Synthesis of lanthanum-doped MCM-48 molecular sieves and its catalytic performance for the oxidation of styrene. J. Phys. Chem. B 2007, 111, 12103-12112. [CrossRef] [PubMed]

10. Li, Y.Z.; Fan, Z.Y.; Shi, J.W.; Liu, Z.Y.; Zhou, J.W.; Shangguan, W.F. Catalytic oxidation of low concentration formaldehyde with the assist of ozone over supported cobalt-manganese composite oxides. J. Mol. Catal. 2014, 1, 60-66.

11. Chou, B.; Tsai, J.L.; Cheng, S. Cu-substituted molecular sieves as liquid phase oxidation catalysts. Microp. Mesop. Mater. 2001, 48, 309-317. [CrossRef]

12. Taran, O.P.; Zagoruiko, A.N.; Ayusheev, A.B.; Yashnik, S.A.; Prihod'Ko, R.V. Cu and Fe-containing ZSM-5 zeolites as catalysts for wet peroxide oxidation of organic contaminants: Reaction kinetics. Res. Chem. Intermed. 2015, 41, 9521-9537. [CrossRef]

13. TOPAS. General Profile and Structure Analysis Software for Powder Diffraction Data, V4.2; Bruker AXS GmbH: Karlsruhe, Germany, 2009. 
14. Cheary, R.W.; Coelho, A. A fundamental parameters approach to X-ray line-profile fitting. J. Appl. Crystallogr. 1992, 25, 109-121. [CrossRef]

15. Liu, W.M.; Hu, Y.Q.; Tu, S.T. Ruthenium supported on active carbon-ceramic sphere as catalysts for catalytic wet air oxidation (CWAO) of resin effluent. J. Hazard. Mater. 2010, 179, 545-551. [CrossRef] [PubMed]

16. Yu, C.Y.; Meng, X.; Chen, G.X.; Zhao, P.Q. Catalytic wet air oxidation of high concentration organic pollutants by upflow packed-bed reactor using a Ru-Ce catalyst derived from $\mathrm{Ru}_{3}(\mathrm{CO})_{12}$ precursor. RSC Adv. 2016, 6, 22633-22638. [CrossRef]

17. Ricq, N.; Barbati, S.; Ambrosio, M. Optimization of the degradation of sewage sludge by wet air oxidation. Study of the reaction mechanism on a cellulose model compound. Analusis 2001, 29, 872-877. [CrossRef]

2018 by the authors. Licensee MDPI, Basel, Switzerland. This article is an open access article distributed under the terms and conditions of the Creative Commons Attribution (CC BY) license (http://creativecommons.org/licenses/by/4.0/). 